\title{
Visual information feedback mechanism and attitudinal prioritisation method for group decision making with triangular fuzzy complementary preference relations
}

\author{
Jian $\mathrm{Wu}^{\mathrm{a}, \mathrm{b}}$, Francisco Chiclana $\mathrm{a}^{\mathrm{b}, \mathrm{c}}$ \\ ${ }^{a}$ School of Economics and Management, Zhejiang Normal University, Jinhua, Zhejiang, China \\ ${ }^{b}$ Centre for Computational Intelligence, Faculty of Technology, De Montfort University, Leicester, UK \\ ${ }^{c}$ DMU Interdisciplinary Group in Intelligent Transport Systems, Faculty of Technology, De Montfort University, \\ Leicester, UK
}

\begin{abstract}
A visual information feedback mechanism for group decision making (GDM) problems with triangular fuzzy complementary preference relations (TFCPRs) is investigated. The concepts of similarity degree (SD) between two experts as well as the proximity degree (PD) between an expert and the rest of experts in the group are developed for TFCPRs. The consensus level (CL) is defined by combining $\mathrm{SD}$ and $\mathrm{PD}$, and a feedback mechanism is proposed to identify experts, alternatives and corresponding preference values that contribute less to consensus. The novelty of this feedback mechanism is that it will provide each expert with visual representations of his/her consensus status to easily 'see' his/her consensus position within the group as well as to identify the alternatives and preference values that he/she should be reconsidered for changing in the subsequent consensus round. The feedback mechanism also includes individualised recommendation to those identified experts on changing their identified preference values and visual graphical simulation of future consensus status if the recommended values were to be implemented. Based on the continuous ordered weighted average (COWA) operator, the triangular fuzzy COWA (TF-COWA) operator is defined, and a novel attitudinal expected score function for TFCPRs is developed. The advantage of this function is that the alternatives are ranked by taking into account the attitudinal character of the group of experts or its moderator if applicable. Additionally, a ranking sensitivity analysis of the attitudinal expected score function with respect to the attitudinal parameter is provided.
\end{abstract}

Keywords: Group decisions making, Visual information feedback mechanism, Triangular fuzzy complementary preference relations, Consensus, Risk attitude

\section{Introduction}

Preference relations, also known as pairwise comparison matrices, are a popular and powerful method to model experts' preferences in group decision making (GDM) problems. The main advantage of preference relations is that individuals can focus exclusively on two alternatives at a time, which

Email addresses: jyajian@163.com (Jian Wu), chiclana@dmu.ac.uk (Francisco Chiclana) 
facilitates the expression of their opinions [29] and then makes them more accurate than non-pairwise methods [45].

In classical decision making systems, given an ordered pair of alternatives, the set of numerical values $\{1,0.5,0\}$, or its equivalent $\{1,0,-1\}[21,22]$, is used to represent the following three preference states: (i) the first alternative is preferred to the second one; (ii) both alternatives are considered equally preferred (indifference); and (iii) the second alternative is preferred to the first one, respectively. This numeric discrimination model of preferences is the simplest possible and it proves insufficient in decision making situations where the implementation of 'intensity of preference' between alternatives is necessary [13].

The concept of fuzzy set when applied to a classical relation leads to the concept of a fuzzy relation, which in turn allows the implementation of intensity of preferences [73]. In this approach, the numeric scale used to evaluate intensity of preferences is the continuum set $[0,1][2,10,12,32,36,48,56,61,64]$. An alternative and isomorphic numeric scale [11], [1/9,9], used when the valuations are measured by a ratio scale, rather than a difference scale as in the previous case, is also possible and widely studied in literature $[4,41,47,65]$. Interval extensions of these two types of numeric preference relations have also been developed $[5,19,24,39,40,52,55,57,60]$.

Subjectivity, imprecision and vagueness in the articulation of opinions pervade real world decision applications, and individuals usually find it difficult to evaluate their preference using exact numbers [73]. Individuals might feel more comfortable using words by means of linguistic labels or terms to articulate their preferences [74]. Furthermore, humans exhibit a remarkable capability to manipulate perceptions and other characteristics of physical and mental objects, without any exact numerical measurements and complex computations [17, 42, 43, 46, 58, 69, 75].

The main two methodologies to represent linguistic preference relations (LPRs) in decision making are [27]: (i) the cardinal representation model based on the use of fuzzy sets and their associated membership functions, which are mathematically processed using Zadeh's extension principle [73]; and (ii) the ordinal representation model by means of the 2-tuples methodology [28, 30, 66]. Although the later representation is able to capture some of the linguistic information to model, it is in fact processed using mathematical tools that are not appropriate for ordinal information but for information provided using a difference or ratio scale. Evidence of this is that the ordinal linguistic model is mathematically equivalent to the cardinal approach with fuzzy sets represented using a representative element of its membership function, and example of which is the centroid. Therefore, the uncertainty nature of the information is lost in the ordinal linguistic computational model. Furthermore, the linguistic cardinal approach is richer than the ordinal linguistic approach, not only because it has the latter one as a particular case, but also because it provides a more flexible tool for GDM with LPRs in which: (1) the experts and/or moderator attitudinal character can be implemented in the decision making process; and (2) different types of fuzzy sets are possible to be used depending on the type and intensity of 
the imprecision and vagueness contained in the linguistic information to model. In particular, the use of triangular fuzzy sets to model linguistic information, which leads to the so-called triangular fuzzy complementary preference relations (TFCPRs) $[15,49,51,53,54,63]$ are worth investigating because they extend both numeric preference relations and interval-valued preference relations. This paper focuses on the use of this type of linguistic preference relation to formulate a framework for GDM problems.

GDM problems generally involve situations of conflict among its experts, and therefore it is preferable that the set of experts reach consensus before applying a selection process to derive the decision solution. There are two basic consensus models in GDM: the static consensus models [6, 23, 37, 70, 76] and the interactive consensus models $[3,9,33,34,62]$. The former does not implement any type of feedback mechanism to advice experts on how to change their preferences in order to achieve a higher consensus level while the later does. Existing interactive consensus models methodology relies on the imposition to decision makers (DM) of changes in their opinion when consensus is below a threshold value. However, in practice, it is up to the decision maker to implement or not the recommendations given to him/her [18]. A more reasonable and suitable policy should rest on this premise and, consequently, it would allow the DM to revisit his/her evaluations using appropriate and meaningful consensus information representation. Therefore, the aim of this article is to propose a visual information feedback mechanism for GDM to provide each expert with visual representations of his/her consensus status to easily 'see' his/her consensus position within the group as well as to identify the alternatives and preference values that he/she should be reconsidered for changing in the subsequent consensus round. The feedback mechanism also includes individualised recommendation to those identified experts on changing their identified preference values and visual graphical simulation of future consensus status if the recommended values were to be implemented. To achieve this, a first objective of this paper is to extend Hsu and Chen's similarity degree (SD) [35] to the case of TFCPRs to measure, in the unit interval, how close two individual experts are. The proximity of an expert with respect to the whole group of experts is also measured, resulting in individual proximity degree (PDs). Consensus level (CLs) is defined as a linear combination of SDs withPDs, and all will be defined at the three different levels of a preference relation: the pairs of alternatives, the alternatives and the whole set of alternatives.

An additional limitation of the above consensus models is that they do not take into account the risk attitude of decision makers in the prioritisation process. Therefore, they are not rich enough to capture all the information contained in TFCPRs. Therefore, a second objective of this paper is to define a new prioritisation method for TFCPRs. In the case of interval-valued preference relations, we can find proposals based on their transformation to numeric preference relations by the continuous interval argument ordered weighted average (C-OWA) operator [67, 71, 72]. In [59], the interval-valued intuitionistic fuzzy COWA (IVIF-COWA) operator, which is also used to derive numeric preference 
relations, is investigated. The advantage of these methods is that ranking of the alternatives is obtained by taking into account the expert's attitudinal character. Recall that interval numbers are particular case of triangular fuzzy numbers, and therefore this link allows us to motivate the definitions of the triangular fuzzy COWA (TF-COWA) operator and its associated attitudinal expected score function. The novelty of this score function is that it can be used to derive a numeric preference relation from a TFCPR, from which easily derive a final ranking of the alternatives. Furthermore, a ranking sensitivity analysis of the attitudinal expected score function with respect to the attitudinal parameter is provided.

Summarising, this paper aims to develop a novel visual information feedback mechanism and attitudinal prioritisation method for GDM problems with TFCPRs. The rest of paper is set out as follows: Section 2 focuses on the development of similarity and proximity degrees for TFCPRs. In Section 3, the level of consensus for TFCPRs is proposed, and a visual information feedback mechanism to increase the level of consensus is investigated. Section 4 presents the TF-COWA operator to derive a fuzzy preference relation (FPR) from a TFCPR. It also presents a ranking method and its corresponding sensitivity analysis with respect to the attitudinal parameter. An analysis of the proposed GDM model highlighting the main differences with respect to existing GDM models in literature is given in Section 5. Finally, conclusions are drawn in Section 6.

\section{Similarity and proximity degrees of triangular fuzzy complementary preference rela- tions}

A fuzzy subset $\widetilde{A}$ of $\mathbb{R}$ is called a triangular fuzzy number (TFN) when its membership function $\mu_{\widetilde{A}}(x): \mathbb{R} \rightarrow[0,1]$ is $[38]:$

$$
\mu_{\widetilde{A}}(x)= \begin{cases}0, & x<a \\ \frac{x-a}{b-a}, & a<x \leq b \\ \frac{c-x}{c-b}, & b \leq x<c \\ 0, & x>c\end{cases}
$$

A TFN is shortly represented as $\widetilde{A}=(a, b, c)$, with $a$ and $c$ known as the lower and upper bounds, respectively, while $b$ is known as its modal value. When the TFN $\widetilde{A}$ is symmetrical, i.e. when $b=(a+c) / 2$, then $b$ is also its centroid. Numeric preferences are usually mapped to values in the domain $[0,1]$, therefore this is also the domain we will be using in the rest of the paper.

The Representation Theorem of fuzzy sets [73] provides an alternative and convenient way to define fuzzy sets via their corresponding family of crisp $\alpha$-cut sets. The $\alpha$-cut set of a fuzzy set $\widetilde{A}$ is defined as

$$
\widetilde{A}^{\alpha}=\left\{x \in X \mid \mu_{\widetilde{A}}(x) \geq \alpha\right\}
$$


The set of crisp sets $\left\{\widetilde{A}^{\alpha} \mid 0<\alpha \leq 1\right\}$ is said to be a representation of the fuzzy set $A$. Indeed, the fuzzy set $A$ can be represented as

$$
\widetilde{A}=\underset{0<\alpha \leq 1}{\cup} \alpha \widetilde{A}^{\alpha}
$$

with membership function

$$
\mu_{\widetilde{A}}(x)=\underset{\alpha: x \in \widetilde{A}^{\alpha}}{\vee} \alpha,
$$

where $\vee$ is the maximum operator. For TFNs we have:

$$
\widetilde{A}^{\alpha}=[a+\alpha \cdot(b-a), c-\alpha \cdot(c-b)], \forall \alpha \in[0,1]
$$

Fuzzy sets arithmetic is carried out using Zadeh's extension principle [74]. The extension principle provides the methodology to extend real functions, i.e. functions whose inputs are real numbers, $f\left(x_{1}, x_{2}\right)$, into fuzzy functions, i.e. functions whose inputs are fuzzy sets, $f\left(\widetilde{A}_{1}, \widetilde{A}_{2}\right)$. The extension principle states that $f\left(\widetilde{A}_{1}, \widetilde{A}_{2}\right)$ is a fuzzy set with membership function $\mu_{f\left(\widetilde{A}_{1}, \widetilde{A}_{2}\right)}:[0,1] \rightarrow[0,1]$ :

$$
\mu_{f\left(\widetilde{A}_{1}, \widetilde{A}_{2}\right)}(y)=\sup _{\substack{f\left(x_{1}, x_{2}\right)=y \\ x_{1}, x_{2} \in[0,1]}}\left[\mu_{\widetilde{A}_{1}}\left(x_{1}\right) \wedge \mu_{\widetilde{A}_{2}}\left(x_{2}\right)\right],
$$

where $\wedge$ is the minimum operator. For TFNs, $\widetilde{A}_{1}=\left(a_{1}, b_{1}, c_{1}\right)$ and $\widetilde{A}_{2}=\left(a_{2}, b_{2}, c_{2}\right)$, we have [38]:

1) $\widetilde{A}_{1} \oplus \widetilde{A}_{2}=\left(a_{1}, b_{1}, c_{1}\right) \oplus\left(a_{2}, b_{2}, c_{2}\right)=\left(a_{1}+a_{2}, b_{1}+b_{2}, c_{1}+c_{2}\right)$.

2) $\widetilde{A}_{1} \ominus \widetilde{A}_{2}=\left(a_{1}, b_{1}, c_{1}\right) \ominus\left(a_{2}, b_{2}, c_{2}\right)=\left(a_{1}-c_{2}, b_{1}-b_{2}, c_{1}-a_{2}\right)$.

3) $\widetilde{A}_{1} \otimes \widetilde{A}_{2}=\left(a_{1}, b_{1}, c_{1}\right) \otimes\left(a_{2}, b_{2}, c_{2}\right) \approx\left(a_{1} \cdot a_{2}, b_{1} \cdot b_{2}, c_{1} \cdot c_{2}\right)$.

4) $\widetilde{A}_{1} / \widetilde{A}_{2}=\left(a_{1}, b_{1}, c_{1}\right) /\left(a_{2}, b_{2}, c_{2}\right) \approx\left(a_{1} / c_{2}, b_{1} / b_{2}, c_{1} / a_{2}\right)$.

5) $1 / \widetilde{A}=1 /(a, b, c) \approx(1 / c, 1 / b, 1 / a)$.

A preference relation on a set of alternatives $X=\left\{x_{1}, x_{2}, \ldots, x_{n}\right\}$ with elements being TFNs, $\widetilde{P}=\left(\widetilde{p}_{i j}\right)_{n \times n}$ and $\widetilde{p}_{i j}=\left(a_{i j}, b_{i j}, c_{i j}\right)$, is called a triangular fuzzy complementary preference relation (TFCPR) if the following property holds [38]:

$$
a_{i j}+c_{j i}=b_{i j}+b_{j i}=c_{i j}+a_{j i}=1, \forall i, j=1,2, \ldots n,
$$

\subsection{Similarity degrees}

In the following, we provide the formal definition of distance and similarity functions as given in $[16]$ :

Definition 1 (Distance). Let $A$ be a set. A function $d: A \times A \longrightarrow \mathbb{R}$ is called a distance (or dissimilarity) on $A$ if, for all $x, y \in A$, there hold

1. $d(x, y) \geq 0$ (non-negativity) 
2. $d(x, y)=d(y, x)$ (symmetry)

3. $d(x, x)=0$ (reflexivity)

Definition 2 (Similarity). Let $A$ be a set. A function $s: A \times A \longrightarrow \mathbb{R}$ is called a similarity on $A$ if $s$ is non-negative, symmetric, and if $s(x, y) \leq s(x, x)$ holds for all $x, y \in A$, with equality if and only if $x=y$.

The main transforms between a distance $d$ and a similarity $s$ bounded by 1 are [16]:

$$
d=1-s ; d=\frac{1-s}{s} ; d=\sqrt{1-s} ; d=\sqrt{2 \cdot\left(1-s^{2}\right)} ; d=\arccos s ; d=-\ln s
$$

In this paper, we use the first transform to go from a distance function to a similarity function.

Given two TFN, $\widetilde{A}_{1}=\left(a_{1}, b_{1}, c_{1}\right)$ and $\widetilde{A}_{2}=\left(a_{2}, b_{2}, c_{2}\right)$, their distance $d\left(\widetilde{A}_{1}, \widetilde{A}_{2}\right)$ can be defined as follows [77]:

$$
d\left(\widetilde{A}_{1}, \widetilde{A}_{2}\right)=\frac{\left|a_{1}-a_{2}\right|+\left|b_{1}-b_{2}\right|+\left|c_{1}-c_{2}\right|}{3}
$$

Therefore, the similarity between two TFNs, $\widetilde{A}_{1}=\left(a_{1}, b_{1}, c_{1}\right)$ and $\widetilde{A}_{2}=\left(a_{2}, b_{2}, c_{2}\right)$, will be

$$
s\left(\widetilde{A}_{1}, \widetilde{A}_{2}\right)=1-\frac{\left|a_{1}-a_{2}\right|+\left|b_{1}-b_{2}\right|+\left|c_{1}-c_{2}\right|}{3}
$$

In the following, the similarity degree between two experts using TFCPRs is introduced:

Definition 3. Let $P^{h}=\left(p_{i k}^{h}\right)$ and $P^{l}=\left(p_{i k}^{l}\right)$ be two TFCPRs on a set of alternatives $X$ provided by two experts $E_{h}$ and $E_{l}$, respectively. Then, the similarity degree between experts $E_{h}$ and $E_{l}$ on the pair of alternatives $\left(x_{i}, x_{k}\right), S D_{i k}^{h l}$, is :

$$
S D_{i k}^{h l}=S D\left(p_{i k}^{h}, p_{i k}^{l}\right)=1-d\left(p_{i k}^{h}, p_{i k}^{l}\right)
$$

Denoting $p_{i k}^{h}=\left(a_{i k}^{h}, b_{i k}^{h}, c_{i k}^{h}\right)$ and $p_{i k}^{l}=\left(a_{i k}^{l}, b_{i k}^{l}, c_{i k}^{l}\right)$, then:

$$
S D_{i k}^{h l}=1-\frac{\left|a_{i k}^{h}-a_{i k}^{l}\right|+\left|b_{i k}^{h}-b_{i k}^{l}\right|+\left|c_{i k}^{h}-c_{i k}^{l}\right|}{3}
$$

Note that $S D_{i k}^{h l}=1$ implies $\left|a_{i k}^{h}-a_{i k}^{l}\right|=\left|b_{i k}^{h}-b_{i k}^{l}\right|=\left|c_{i k}^{h}-c_{i k}^{l}\right|=0$ and therefore $p_{i k}^{h}=p_{i k}^{l}$. Therefore, we have the following interpretation: the higher the value of $S D_{i k}^{h l}$, the more similar $p_{i k}^{h}$ and $p_{i k}^{l}$ are.

Definition 4. The similarity degree between experts $E_{h}$ and $E_{l}$ on the alternative $x_{i}, S D_{i}^{h l}$, is:

$$
S D_{i}^{h l}=S D\left(p_{i}^{h}, p_{i}^{l}\right)=\frac{\sum_{k=1}^{n} S D\left(p_{i k}^{h}, p_{i k}^{l}\right)}{n}
$$

As above, when $S D_{i}^{h l}=1$, then both experts provide the same linguistic valuation for pairs of alternatives involving $x_{i}$. Thus, the higher the value of $S D_{i}^{h l}$, the more similar the experts' preferences are on the alternative $x_{i}$. 
Definition 5. The similarity degree between experts $E_{h}$ and $E_{l}$ on the whole set of alternatives $X$, $S D^{h l}$ is:

$$
S D^{h l}=S D\left(P^{h}, P^{l}\right)=\frac{\sum_{i=1}^{n} \sum_{k=1}^{n} S D\left(p_{i k}^{h}, p_{i k}^{l}\right)}{n^{2}}
$$

Clearly, $S D^{h l}=1$ means that both experts provide identical TFCPRs, and we can interpret this similarity degree as follows: the higher the value $S D^{h l}$, the closer both experts are in their preferences on the set of alternatives.

We have the following result:

Proposition 1. Let $\left\{P^{1}, P^{2}, \ldots, P^{m}\right\}$ be a set of TFCPRs, then we have

1) Reflexivity: $S D^{h h}=1 \forall h$

2) Symmetry: $S D^{h l}=S D^{l h} \forall h, l$

3) Transitivity: $S D^{h l}=S D^{l t}=1 \Rightarrow S D^{h t}=1$

The similarity degrees of an expert with the rest of the group of experts at the three different levels of a relation are defined next:

Level 1. Similarity degree on pairs of alternatives. The similarity degree of an expert $e_{h}$ on the pair of alternatives $\left(x_{i}, x_{k}\right)$ to the rest of experts in the group is calculated as:

$$
S P A_{i k}^{h}=\frac{\sum_{l=1, l \neq h}^{m} S D_{i k}^{h l}}{m-1}
$$

Level 2. Similarity degree on alternatives. The similarity degree of an expert $e_{h}$ on alternative $x_{i}$ to the rest of experts in the group is calculated as:

$$
S A_{i}^{h}=\frac{\sum_{k=1}^{n} S P A_{i k}^{h}}{n}
$$

Level 3. Similarity degree on the preference relation. The similarity degree of an expert $e_{h}$ on the whole set of alternative $X$ to the rest of experts in the group is calculated as:

$$
S D^{h}=\frac{\sum_{i=1}^{n} S A_{i}^{h}}{n}
$$

Finally, each expert in the group decision making problem can be associated a relative (normalised) importance degree based on the similarity degrees at level 3 computed above, which we obviously refer to as the relative similarity degree of an expert:

$$
R S D^{h}=\frac{S D^{h}}{\sum_{l=1}^{m} S D^{l}}
$$

Obviously, these relative importance degrees could be different to particular importance weights the experts in the group are assigned before they provide their linguistic information on the set of alternatives. Our methodology is to implement both importance degrees in the computation of consensus 
to reflect the actual position of experts in the group as a collective. This will be developed in the following subsection. Next we provide a simple GDM example to illustrate the computation of the similarity degrees al the three levels of a relation and the final relative similarity degrees of the experts in the group.

Example 1. Suppose four experts $\left\{e_{1}, e_{2}, e_{3}, e_{4}\right\}$ with associated importance degrees $I D=$ $(0.2,0.1,0.4,0.3)^{T}$, are asked to provide their preference on a set of four alternatives $\left\{x_{1}, x_{2}, x_{3}, x_{4}\right\}$, being their linguistic preferences modelled via the following TFCPRs:

$$
\begin{aligned}
& P^{\mathbf{1}}=\left(\begin{array}{cccc}
- & (0.3,0.4,0.5) & (0.4,0.5,0.6) & (0.5,0.6,0.7) \\
(0.5,0.6,0.7) & - & (0.4,0.5,0.6) & (0.3,0.4,0.5) \\
(0.4,0.5,0.6) & (0.4,0.5,0.6) & - & (0.5,0.6,0.7) \\
(0.3,0.4,0.5) & (0.5,0.6,0.7) & (0.3,0.4,0.5) & -
\end{array}\right) \\
& P^{\mathbf{2}}=\left(\begin{array}{cccc}
(0.4,0.5,0.6) & - & (0.5,0.6,0.7) & (0.5,0.6,0.7) \\
(0.6,0.7,0.8) & (0.3,0.4,0.5) & - & (0.1,0.2,0.3) \\
(0.5,0.6,0.7) & (0.3,0.4,0.5) & (0.7,0.8,0.9) & -
\end{array}\right) \\
& P^{\mathbf{3}}=\left(\begin{array}{ccccc}
- & (0.4,0.5,0.6) & (0.2,0.3,0.4) & (0.3,0.4,0.5) \\
(0.3,0.4,0.5) & - & (0.5,0.6,0.7) & (0.2,0.3,0.4) \\
(0.4,0.5,0.6) & (0.3,0.4,0.5) & - & (0.4,0.5,0.6) \\
(0.2,0.3,0.4) & (0.6,0.7,0.8) & (0.4,0.5,0.6) & -
\end{array}\right) \\
& P^{4}=\left(\begin{array}{llll}
(0.4,0.5,0.6) & - & (0.6,0.7,0.8) & (0.2,0.3,0.4) \\
(0.3,0.4,0.5) & (0.2,0.3,0.4) & - & (0.3,0.4,0.5) \\
(0.3,0.4,0.5) & (0.6,0.7,0.8) & (0.5,0.6,0.7) & -
\end{array}\right)
\end{aligned}
$$

I) The similarity degree on pairs of alternatives for each expert are:

$$
\begin{aligned}
S P A^{1}= & \left(\begin{array}{llll}
1.000 & 0.867 & 0.900 & 0.900 \\
0.867 & 1.000 & 0.867 & 0.867 \\
0.900 & 0.867 & 1.000 & 0.767 \\
0.900 & 0.867 & 0.767 & 1.000
\end{array}\right) ; S P A^{2}=\left(\begin{array}{cccc}
1.000 & 0.933 & 0.767 & 0.767 \\
0.933 & 1.000 & 0.933 & 0.733 \\
0.767 & 0.933 & 1.000 & 0.700 \\
0.767 & 0.733 & 0.700 & 1.000
\end{array}\right) \\
S P A^{3} & =\left(\begin{array}{llll}
1.000 & 0.867 & 0.900 & 0.833 \\
0.867 & 1.000 & 0.933 & 0.867 \\
0.900 & 0.933 & 1.000 & 0.833 \\
0.833 & 0.867 & 0.833 & 1.000
\end{array}\right) ; S P A^{4}=\left(\begin{array}{llll}
1.000 & 0.933 & 0.833 & 0.900 \\
0.933 & 1.000 & 0.867 & 0.867 \\
0.833 & 0.867 & 1.000 & 0.833 \\
0.900 & 0.867 & 0.833 & 1.000
\end{array}\right)
\end{aligned}
$$


II) The similarity degrees on alternatives for each expert are:

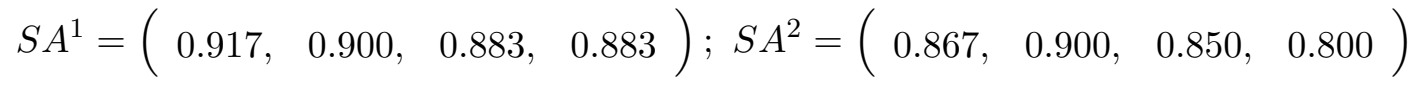

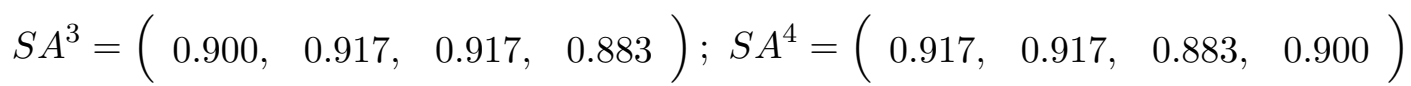

III) The similarity degrees on the set of alternatives for each expert are:

$$
S D^{1}=0.896 ; S D^{2}=0.854 ; S D^{3}=0.904 ; S D^{4}=0.904 .
$$

IV) The relative group similarity degrees for each expert are:

$$
R S D^{1}=0.252 ; R S D^{2}=0.240 ; R S D^{3}=0.254 ; R S D^{4}=0.254 .
$$

\subsection{Proximity degrees}

In a GDM problem, there are two possible scenarios: the importance degree of experts is known beforehand or completely unknown. In the first scenario, the moderator (decision maker) assigns the importance degree of experts according to their experience and reputation, etc. For example, in the process of selecting a key supplier, the purchasing activity is usually carried out by group experts, who may come from different departments: purchasing department, finance department, and quality inspection department. In this case, the general manager usually assigns the highest importance degree to the expert from the purchasing department because of his/her experience or responsibility. In the second scenario, when no explicit importance degrees are provided or associated to the experts, we make note that experts might not necessarily be perceived and treated as equally important. This is specially true, for instance, once the experts have provided information on the particular matter to solve, in which case this information can be used as a mean to discriminate them as not equally important. In these cases, it may be reasonable to compute importance degree of experts by defining indexes based on specific problem context criteria such as consensus or consistency as done in $[12,60,61]$.

The proximity degrees measure the similarity between individual experts' opinions and the collective opinion for the group of experts. The aggregation of individual opinions will be weighted using a weight vector whose elements are a linear combination of the importance degree of individuals before the decision making process and the relative similarity degrees computed based on the information they provided as per the previous subsection. This is elaborated next:

(1) Experts weighting vector

$$
W=\eta \cdot I D+(1-\eta) \cdot R S D
$$

If $\eta>0.5$, then the group/moderator values higher the a priori importance degrees of the experts than their a posteriori relative similarity degrees. Obviously, for homogeneous GDM problems the value $\eta=0$ applies. 
(2) The collective TFCPR, $P=\left(p_{i k}\right)_{n \times n}$, is computed as follows:

$$
p_{i k}=w^{1} \otimes p_{i k}^{1} \oplus w^{2} \otimes p_{i k}^{2} \oplus \cdots \oplus w^{m} \otimes p_{i k}^{m}
$$

Example 2. (Example 1 Continuation) Assuming a value of $\eta=0.5$ we have the following weighting vector

$$
W=0.5 * I D+0.5 * R S D=(0.22,0.17,0.33,0.28)^{T}
$$

and the collective TFCPR is

$$
P=\left(\begin{array}{cccc}
- & (0.41,0.51,0.61) & (0.39,0.49,0.59) & (0.50,0.60,0.70) \\
(0.39,0.49,0.59) & - & (0.51,0.61,0.71) & (0.27,0.37,0.47) \\
(0.41,0.51,0.61) & (0.39,0.49,0.59) & - & (0.34,0.44,0.54) \\
(0.30,0.40,0.50) & (0.53,0.63,0.73) & (0.46,0.56,0.66) & -
\end{array}\right)
$$

Once the collective TFCPR is obtained, we compute the proximity measures for each expert at the three different levels of a relation:

Level 1. Proximity degree on pairs of alternatives. The proximity degree of an expert $e_{h}$ to the group on the pair of alternatives $\left(x_{i}, x_{k}\right)$ is

$$
P P A_{i k}^{h}=S D\left(p_{i k}^{h}, p_{i k}\right)
$$

Level 2. Proximity degree on alternatives. The proximity degree of an expert $e_{h}$ to the group on the alternative $x_{i}$ is

$$
P A_{i}^{h}=\frac{\sum_{k=1}^{n} P P A_{i k}^{h}}{n}
$$

Level 3. Proximity degree on the preference relation. The proximity degree of an expert $e_{h}$ to the group on the set of alternatives $X$ is

$$
P D^{h}=\frac{\sum_{i=1}^{n} P A_{i}^{h}}{n}
$$

Example 3. (Example 1 Continuation) Proximity degrees computation.

I) The proximity degree on pairs of alternatives for each expert are:

$$
P P A^{1}=\left(\begin{array}{cccc}
1.000 & 0.889 & 0.994 & 0.999 \\
0.889 & 1.000 & 0.894 & 0.973 \\
0.994 & 0.894 & 1.000 & 0.843 \\
0.999 & 0.973 & 0.843 & 1.000
\end{array}\right) ; P P A^{2}=\left(\begin{array}{cccc}
1.000 & 0.989 & 0.806 & 0.801 \\
0.989 & 1.000 & 0.994 & 0.773 \\
0.806 & 0.994 & 1.000 & 0.757 \\
0.801 & 0.773 & 0.757 & 1.000
\end{array}\right)
$$




$$
P P A^{3}=\left(\begin{array}{cccc}
1.000 & 0.911 & 0.994 & 0.899 \\
0.911 & 1.000 & 0.994 & 0.927 \\
0.994 & 0.994 & 1.000 & 0.943 \\
0.899 & 0.927 & 0.943 & 1.000
\end{array}\right) ; P P A^{4}=\left(\begin{array}{cccc}
1.000 & 0.989 & 0.894 & 0.999 \\
0.989 & 1.000 & 0.906 & 0.927 \\
0.894 & 0.906 & 1.000 & 0.957 \\
0.999 & 0.927 & 0.957 & 1.000
\end{array}\right)
$$

II) The proximity degrees on alternatives for each expert are:

$$
\begin{aligned}
& P A^{1}=\left(\begin{array}{llll}
0.971, & 0.939, & 0.933, & 0.954
\end{array}\right) ; P A^{2}=\left(\begin{array}{llll}
0.899, & 0.939, & 0.889, & 0.833
\end{array}\right)
\end{aligned}
$$

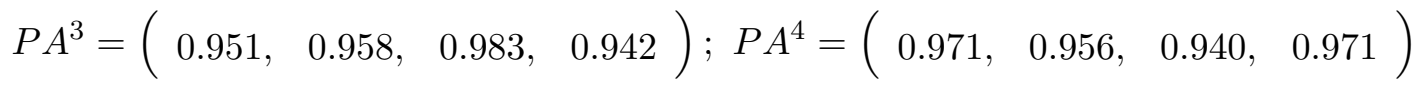

III) The proximity degrees on the relation for each expert are:

$$
P D^{1}=0.949 ; P D^{2}=0.890 ; P D^{3}=0.958 ; P D^{4}=0.959 \text {. }
$$

\section{Consensus model with visual information feedback mechanism for GDM with TFCPRs}

In a GDM, it is desirable that the group of experts achieve a high consensus level among their preferences. Consensus is defined as agreement among experts regarding the feasible alternatives, and therefore its definition definition is based on the concept of similarity between their opinions (preferences). Both similarity degree (SD) and the proximity degree (PD) convey the concept of similarity between experts in a group: the first one between pairs of individual experts and the second one between an individual expert and the rest of experts in the group. Thus, both degrees could/should be used in measuring the level of consensus within a group of experts regarding the set of feasible alternatives in GDM. The simplest of the combinations is the linear one, and it is here used to propose the following definitions of the consensus level (CL) associated to each expert of the group at the three different levels of a relation:

Level 1. Consensus level on the pairs of alternatives (CLPA). The consensus level of an expert $e_{h}$ on the pair of alternatives $\left(x_{i}, x_{k}\right)$ is

$$
C L P A_{i k}^{h}=\psi \cdot S P A_{i k}^{h}+(1-\psi) \cdot P P A_{i k}^{h}
$$

Level 2. Consensus level on the alternatives (CLA). The consensus level of an expert $e_{h}$ on the alternative $x_{i}$ is

$$
C L A_{i}^{h}=\psi \cdot S A_{i}^{h}+(1-\psi) \cdot P A_{i}^{h}
$$

Level 3. Consensus level on the relation $(C L)$. The consensus level (CL) $e_{h}$ on the set of alternatives $X$ is

$$
C L^{h}=\psi \cdot S D^{h}+(1-\psi) \cdot P D^{h}
$$


with $\psi \in[0,1]$ a parameter to control the weight of both similarity and proximity criteria. Unless there are specific reasons to prefer one index to the other one, the value to assume for the weighting parameter $\psi$ should be 0.5 , as it is assumed in the example below.

Example 4. (Example 1 Continuation) Consensus levels computation. Setting $\psi$ at 0.5, the following consensus levels on the relation are obtained:

$$
C L^{1}=0.922, \quad C L^{2}=0.872, \quad C L^{3}=0.932, \quad C L^{4}=0.932
$$

The only expert with a consensus level below the threshold value is $e_{2}$ and therefore he/she will receive feedback advice on how to change his/her preferences to achieve a higher consensus level.

In practice, it is rare to achieve full and unanimous agreement of all the experts regarding all the feasible alternatives. As a consequence, the consensus threshold value $(\gamma)$ to achieve is usually set to a value lower than 1 . At the same time, the decision output should be acceptable for at least half of the experts, which means that the parameter $\gamma$ should be set to a value no lower than 0.5. If the consensus level is not acceptable, that is, if it is lower than the specified threshold value, the experts are normally invited to discuss their opinions further in an effort to make them closer. To help experts in their discussion, a visual information feedback mechanism is here proposed to provide each expert with visual representations of his/her consensus status to easily 'see' their relative consensus position within the group as well as to identify the alternatives and preference values that he/she should reconsider for changing in the subsequent consensus round. Additionally, the visual feedback mechanism also includes individualised recommendation to those experts that are identified as contributing less to consensus on how to change their identified preference values and a visual graphical simulation of future consensus status if the recommended values were to be implemented. In the light of this visual extra information, an expert can revisit his evaluations and make changes if considered appropriate to achieve a higher consensus level. This consensus model with visual information feedback mechanism process is illustrated in Figure 1.

In the following, a detailed description of the visual feedback methodology is provided. As it was mentioned above, personalised advice to those experts with a consensus level below the acceptable threshold value on how to change their preferences to increase their consensus level are also generated.

\subsection{Visual Information Feedback Mechanism}

The visual information feedback mechanism consists of three stages: firstly, the identification of the triangular fuzzy preference values that should be subject to modification; secondly, the generation of advice on the direction-value of the required change; and, thirdly, the automatic feedback process simulation to show what would happen if experts are to accept the recommended preference values. These three stages are described in detail below: 


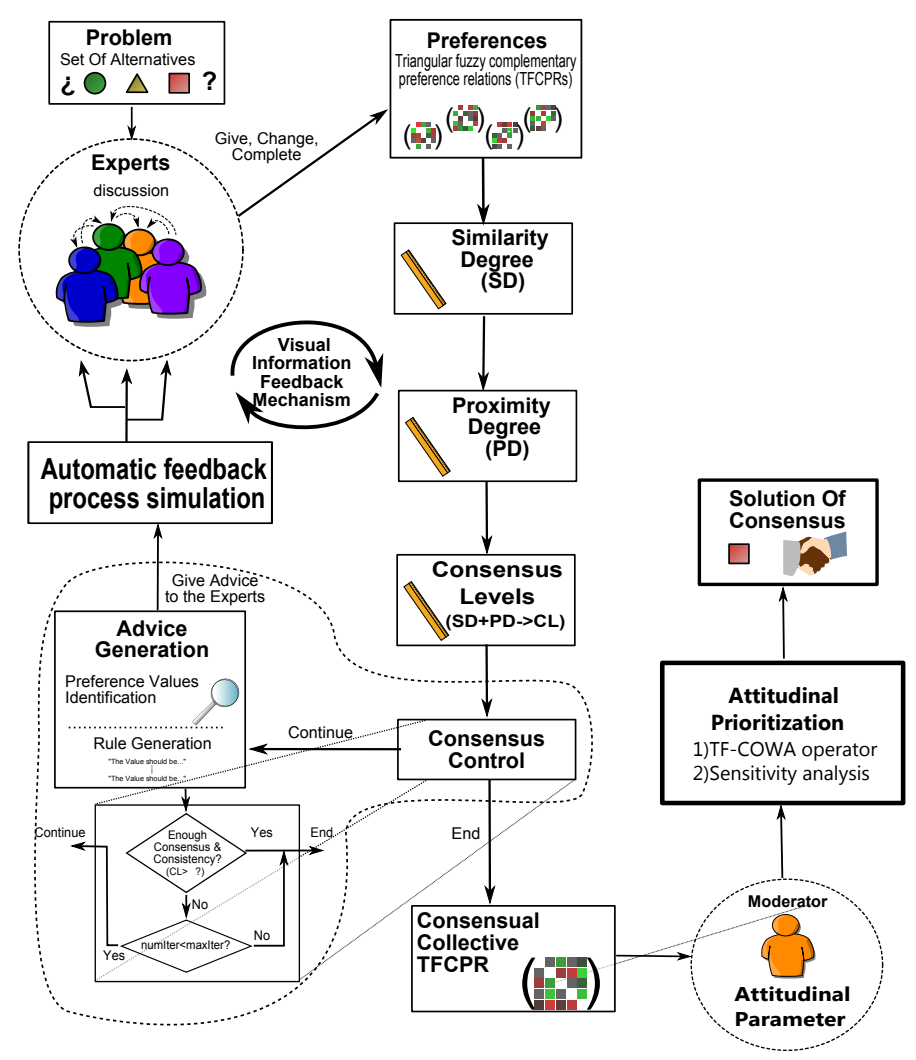

Figure 1: Consensus model with visual information feedback mechanism process

(1) Identification of the Triangular Fuzzy Preference Values: The set of triangular fuzzy preference values that contribute less to reach an acceptable consensus level is identified and presented to the experts using visual graphs as illustrated in Figure 2. Once consensus levels are computed, at the relation level, all experts will receive a visual representation of their consensus status in relation to the threshold value, which can be used to easily identify the experts furthest from the group. Following with Example 4, and using a threshold value $\gamma=0.9$, Figure 2(a) presents a visual representation of all experts consensus level with respect to the threshold value from which expert $e_{2}$ is clearly identified as the only expert contributing less to group consensus. If necessary, individual visual representations of consensus levels of alternatives and pair of alternatives are also provided to each expert to help them identify those alternatives and their associated preference values at the level of pairs of alternatives that contribute less to consensus and, consequently, potential to be reconsidered for changing in the next round of consensus. In Example 4, this is necessary to be done for expert $e_{2}$ whom would receive visual representation at these levels as illustrated in Figure 2(b) and Figure 2(c), respectively. Mathematically, these steps are modelled as follows:

Step 1. The set of experts with consensus levels below the threshold value $\gamma$ is identified:

$$
E C H=\left\{h \mid C L^{h}<\gamma\right\}
$$


Step 2. For experts identified in step 1, those alternatives with a consensus level below $\gamma$ are identified:

$$
A C H=\left\{(h, i) \mid h \in E C H \wedge C L A_{i}^{h}=\psi \cdot S A_{i}^{h}+(1-\psi) \cdot P A_{i}^{h}<\gamma\right\}
$$

Step 3. Finally, the triangular fuzzy preference values for the experts and alternatives identified in steps 1 and 2 that need to be changed are identified:

$$
P A C H=\left\{(h, i, k) \mid(h, i) \in A C H \wedge C L P A_{i k}^{h}=\psi \cdot S P A_{i k}^{h}+(1-\psi) \cdot P P A_{i k}^{h}<\gamma\right\}
$$
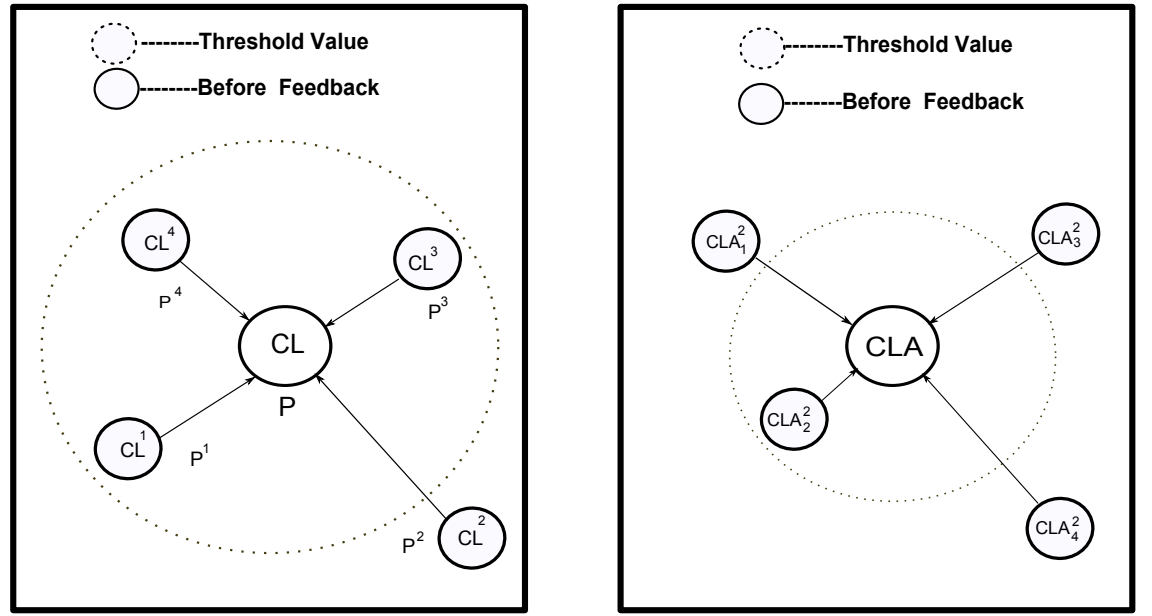

(a) Consensus levels on the relation: $C L^{h}$

(b) Consensus levels on the alternatives for $e_{2}: C L A_{i}^{2}$

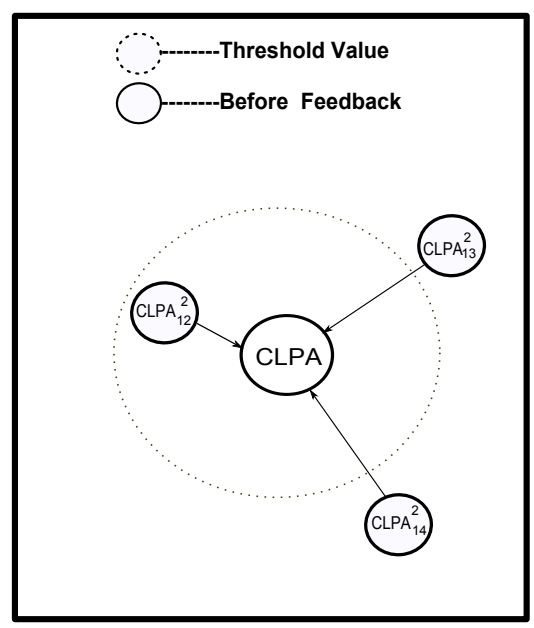

(c) Consensus levels on the pairs of alternatives for $A_{1}$ and $e_{2}: C L P A_{1 j}^{2}$

Figure 2: Visual representation of consensus levels in relation to the consensus threshold value

Example 5. (Example 1 Continuation) The sets of 3-tuple identified as contributing less to consensus are:

$$
P A C H=\{(2,1,3),(2,1,4),(2,2,4),(2,3,1),(2,3,4),(2,4,1),(2,4,2),(2,4,3)\}
$$


(2) Generation of Advice: The feedback mechanism also generates personalised recommendations rules, which will not only tell the experts which preference values they should change, but also provide them with the consensus advice to revisit their evaluation in the light of this extra information.

For all $(h, i, k) \in P A C H$, the following rule is feed backed to the corresponding experts:

"To increase your consensus level $(C L)$, your preference value $p_{i k}^{h}$ should be closer to $\overline{\bar{p}}_{i k}^{h}$."

$$
\overline{\bar{p}}_{i k}^{h}=\psi \cdot \bar{p}_{i k}^{h}+(1-\psi) \cdot p_{i k}
$$

where $\bar{p}_{i k}^{h}=\left(\sum_{l=1, l \neq h}^{m} p_{i k}^{l}\right) /(m-1)$ and $p_{i k}$ the collective preference value. The reciprocity property that the TFCPRs verify implies that when the pair of alternatives $(i, k)$ is identified for change, the pair $(k, i)$ has to be changed accordingly as well.

Example 6. (Example 1 continuation) The recommendations for expert $e_{2}$ are:

- To increase your consensus level (CL), your preference value $p_{13}^{2}$ should be closer to $(0.4,0.5,0.6)$.

- To increase your consensus level (CL), your preference value $p_{31}^{2}$ should be closer to $(0.4,0.5,0.6)$.

- To increase your consensus level (CL), your preference value $p_{14}^{2}$ should be closer to $(0.5,0.6,0.7)$.

- To increase your consensus level (CL), your preference value $p_{41}^{2}$ should be closer to $(0.3,0.4,0.5)$.

- To increase your consensus level (CL), your preference value $p_{24}^{2}$ should be closer to $(0.4,0.5,0.6)$.

- To increase your consensus level (CL), your preference value $p_{42}^{2}$ should be closer to $(0.4,0.5,0.6)$.

- To increase your consensus level (CL), your preference value $p_{34}^{2}$ should be closer to $(0.4,0.5,0.6)$.

- To increase your consensus level (CL), your preference value $p_{43}^{2}$ should be closer to $(0.4,0.5,0.6)$.

(3) Automatic Feedback Process Simulation: A what-if scenario analysis could be run to generate a visual graphical simulation of future consensus status if the recommended values were to be implemented, as shown in Fig 3(a), Fig 3(b) and Fig 3(c). This will provide the decision makers with a clear picture of their actual position within the group, which they can then use to decide upon their actual position or subsequent action. If the advice is implemented, then the consensus level increases as Example 7. Not implementing these advices can lead to the consensus level to remain fixed or to increase at a very low rate, which would make the group consensus threshold value difficult to achieve. To avoid these situation, a maximum number of iterations maxiter can be incorporated in the visual information feedback mechanism following a similar approach of consensus models proposed in $[7,31]$.

Example 7. (Example 1 continuation) After expert $e_{2}$ revisits his/her evaluation and implements the recommended TFNs, a new round of the consensus process is carried out, leading to the following new TFCPRs: 


$$
\begin{array}{r}
P^{\mathbf{1}}=\left(\begin{array}{cccc}
- & (0.3,0.4,0.5) & (0.4,0.5,0.6) & (0.5,0.6,0.7) \\
(0.5,0.6,0.7) & - & (0.4,0.5,0.6) & (0.3,0.4,0.5) \\
(0.4,0.5,0.6) & (0.4,0.5,0.6) & - & (0.5,0.6,0.7) \\
(0.3,0.4,0.5) & (0.5,0.6,0.7) & (0.3,0.4,0.5) & -
\end{array}\right) \\
P^{\mathbf{2}}=\left(\begin{array}{cccc}
- & (0.4,0.5,0.6) & (0.4,0.5,0.6) & (0.5,0.6,0.7) \\
(0.4,0.5,0.6) & - & (0.5,0.6,0.7) & (0.3,0.4,0.5) \\
(0.4,0.5,0.6) & (0.3,0.4,0.5) & - & (0.4,0.5,0.6) \\
(0.3,0.4,0.5) & (0.5,0.6,0.7) & (0.4,0.5,0.6) & -
\end{array}\right) \\
P^{\mathbf{3}}=\left(\begin{array}{cccc}
- & (0.5,0.6,0.7) & (0.4,0.5,0.6) & (0.6,0.7,0.8) \\
(0.3,0.4,0.5) & - & (0.5,0.6,0.7) & (0.2,0.3,0.4) \\
(0.4,0.5,0.6) & (0.3,0.4,0.5) & - & (0.4,0.5,0.6) \\
(0.2,0.3,0.4) & (0.6,0.7,0.8) & (0.4,0.5,0.6) & -
\end{array}\right) \\
P^{4}=\left(\begin{array}{llll}
(0.4,0.5,0.6) & - & (0.6,0.7,0.8) & (0.2,0.3,0.4) \\
(0.3,0.4,0.5) & (0.2,0.3,0.4) & - & (0.3,0.4,0.5) \\
(0.3,0.4,0.5) & (0.6,0.7,0.8) & (0.5,0.6,0.7) & -
\end{array}\right)
\end{array}
$$

The new consensus levels are now:

$$
C L^{1}=0.956, C L^{2}=0.976, C L^{3}=0.961, C L^{4}=0.957
$$

Because all experts are over the minimum consensus threshold value $\gamma=0.9$, the consensual collective TFCPR is computed from which the final solution of consensus will be selected:

$$
P=\left(\begin{array}{cccc}
- & (0.41,0.51,0.61) & (0.43,0.53,0.63) & (0.53,0.63,0.73) \\
(0.39,0.49,0.59) & - & (0.51,0.61,0.71) & (0.24,0.34,0.44) \\
(0.37,0.47,0.57) & (0.39,0.49,0.59) & - & (0.39,0.49,0.59) \\
(0.27,0.37,0.47) & (0.56,0.66,0.76) & (0.41,0.51,0.61) & -
\end{array}\right)
$$

It is worth noting here that quality of the decision making process and quality of the outcome of such decision making process are not necessarily related. Indeed, the visual information feedback mechanism and the selection process here developed assure that the outcome, i.e. the selected alternative, is a solution of consensus but it does not guarantee that is the right decision. Indeed, if the group of experts provide information that is incorrect then the current decision making process will not guarantee that the outcome is correct. It can happen that all but one expert in a group provide similar incorrect information, in which case the consensus support system here developed will 

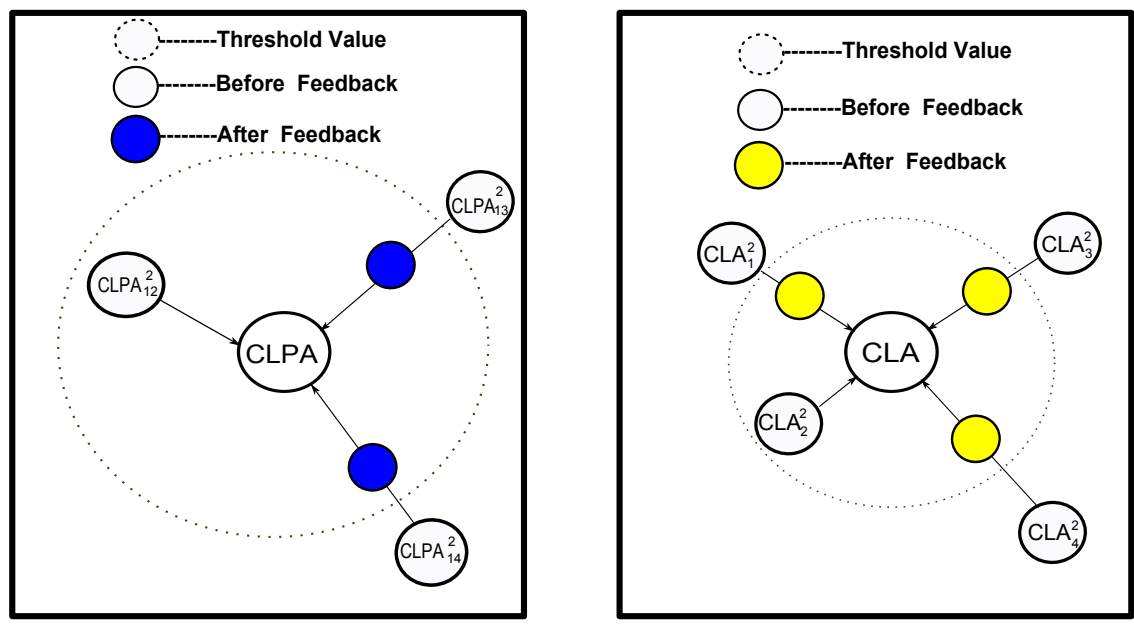

(a) $C L P A_{1 j}^{2}$ before and after $e_{2}$ imple- (b) $C L A_{i}^{2}$ before and after $e_{2}$ implements ments recommended values recommended values

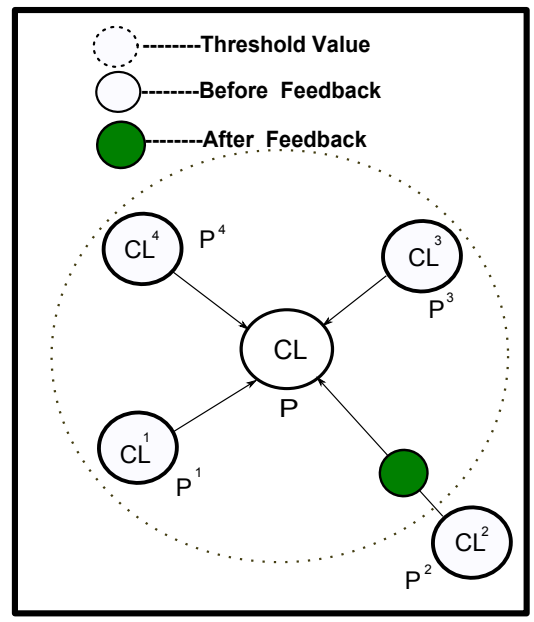

(c) $C L^{h}$ before and after $e_{2}$ implements recommended values

Figure 3: Simulation of consensus before and after recommended values are implemented by expert $e_{2}$

advise the only correct expert to consider changing his/her preferences to make them closer to the rest, although incorrect, experts. Consequently, here the concern is with the quality of the decision making process rather than with the quality of the output. Although being the ultimate goal of any decision making, the quality of the decision outcome is out of our control in that this issue, firstly, resides in the intelligence phase during which the decision problem is detected and, secondly, in the identification and analysis of potential alternative solutions [50]. It is also out of the control of our study the gathering of information on the set of potential alternative solutions to the decision making problem but not its appropriate formal representation in the presence of uncertainty, which was the subject of the previous sections. Summarising, we agree with Marakas [44] in that 'we really do not know whether a decision is a good one until after it is made', and that a post-decision evaluation 
might be necessary carried out to assess the quality of the decision making output.

\section{Attitudinal prioritisation method for TFCPRs}

Once the consensual final collective TFCFPR is computed, a selection process is activated to derive the final solution of consensus to the GDM problem. This is usually done by defining a so-called score function that associated a score or valuation to each one of the alternatives and that is used to produce a final ranking of them. In the flowing, we will develop a score function that is based on the implementation of the decision makers' attitude via the application of the concept of attitudinal character of a basic unit-monotonic (BUM) and the continuous ordered weighted average (COWA) operator introduced in [71]. To do this, in the following we extend the continuous ordered weighted average (COWA) operator to the case in which the argument is a TFN and develop the triangular fuzzy COWA (TF-COWA) operator, which is fundamental in the definition of the TFN attitudinal expected score function.

\subsection{TF-COWA operator and attitudinal expected score function}

Recall that a BUM function is defined as a mapping $Q:[0,1] \rightarrow[0,1]$ such that $Q(0)=0, Q(1)=1$ and $Q(x) \geq Q(y)$ if $x \geq y$. The attitudinal character of a BUM function and the continuous ordered weighted average (COWA) operator were introduced in [71] as follows:

Definition 6 (BUM Attitudinal Character). The attitudinal character of a BUM function, $Q$, is

$$
A-C(Q)=\int_{0}^{1} Q(y) d y
$$

Definition 7 (COWA Operator of Yager). Let $I N T\left(\mathbb{R}^{+}\right)$be the set of all closed subintervals of $\mathbb{R}^{+}$. A continuous ordered weighted average (COWA) operator is a mapping $F_{Q}: \operatorname{INT}\left(\mathbb{R}^{+}\right) \rightarrow \mathbb{R}^{+}$ which has an associated BUM function, $Q$, such that

$$
F_{Q}([a, b])=\int_{0}^{1} \frac{d Q(y)}{d y}[b-y \cdot(b-a)] d y .
$$

Denoting $\lambda=A-C(Q)$ we have

$$
F_{Q}([a, b])=(1-\lambda) \cdot a+\lambda \cdot b
$$

where $\lambda$ is the attitudinal character of the BUM function $Q$. Thus, $F_{Q}([a, b])$ is the weighted average of the end points of the closed interval with attitudinal character parameter, and it is known as the attitudinal expected value of $[a, b]$.

Yager [71] introduced the following desirable properties of the COWA operator:

- If $a_{1} \geq a_{2}$ and $b_{1} \geq b_{2}$, then for all $Q$ we have: $F_{Q}\left(\left[a_{1}, b_{1}\right]\right) \geq F_{Q}\left(\left[a_{2}, b_{2}\right]\right)$. 
- If $Q_{1} \geq Q_{2}$, then $F_{Q_{1}}([a, b]) \geq F_{Q_{2}}([a, b])$.

- For all $Q$, we have $a \leq F_{Q}([a, b]) \leq b$.

In the following, we extend the application of the COWA operator to the case in which our argument is a TFN and develop the triangular fuzzy COWA (TF-COWA) operator.

Definition 8 (TF-COWA Operator). Let $\widetilde{A}=(a, b, c)$ be a TFN and $I N T(\widetilde{A})=\left\{\widetilde{A}^{\alpha}=[a+\alpha\right.$. $(b-a), c-\alpha \cdot(c-b)] \mid 0<\alpha \leq 1\}$ the set of all $\alpha$-cut sets of $\widetilde{A}$. A triangular fuzzy COWA (TF-COWA) operator with associated BUM function $Q$ is a mapping $F_{Q}: I N T(\widetilde{A}) \rightarrow \mathbb{R}^{+}$such that

$$
\begin{aligned}
F_{Q}\left(\widetilde{A}^{\alpha}\right) & =(1-\lambda) \cdot[a+\alpha \cdot(b-a)]+\lambda \cdot[c-\alpha \cdot(c-b)] \\
& =a+\alpha \cdot(b-a)+\lambda \cdot(1-\alpha) \cdot(c-a)
\end{aligned}
$$

The following properties hold:

Proposition 2. Let $\widetilde{A}_{1}=\left(a_{1}, b_{1}, c_{1}\right)$ and $\widetilde{A}_{2}=\left(a_{2}, b_{2}, c_{2}\right)$ be two TFNs with $\alpha$-cut $\widetilde{A}_{1}^{\alpha}$ and $\widetilde{A}_{2}^{\alpha}$, respectively, then

$$
F_{Q}\left(\left(\widetilde{A}_{1}+\widetilde{A}_{2}\right)^{\alpha}\right)=F_{Q}\left(\widetilde{A}_{1}^{\alpha}+\widetilde{A}_{2}^{\alpha}\right)=F_{Q}\left(\widetilde{A}_{1}^{\alpha}\right)+F_{Q}\left(\widetilde{A}_{2}^{\alpha}\right)
$$

Proof. Interval arithmetics implies that $\left(\widetilde{A}_{1}+\widetilde{A}_{2}\right)^{\alpha}=\widetilde{A}_{1}^{\alpha}+\widetilde{A}_{2}^{\alpha}=\left[A_{1}^{L}+A_{2}^{L}, A_{1}^{U}+A_{2}^{U}\right]$, and therefore we have

$$
F_{Q}\left(\widetilde{A}_{1}^{\alpha}+\widetilde{A}_{2}^{\alpha}\right)=(1-\lambda) \cdot\left(A_{1}^{L}+A_{2}^{L}\right)+\lambda \cdot\left(A_{1}^{U}+A_{2}^{U}\right)
$$

Because $F_{Q}\left(\widetilde{A}_{1}^{\alpha}\right)=(1-\lambda) \cdot A_{i}^{L}+\lambda \cdot A_{i}^{U}$ then it is $F_{Q}\left(\widetilde{A}_{1}^{\alpha}+\widetilde{A}_{2}^{\alpha}\right)=F_{Q}\left(\widetilde{A}_{1}^{\alpha}\right)+F_{Q}\left(\widetilde{A}_{2}^{\alpha}\right)$.

Proposition 3. Let $\widetilde{A}_{1}=\left(a_{1}, b_{1}, c_{1}\right)$ and $\widetilde{A}_{2}=\left(a_{2}, b_{2}, c_{2}\right)$ be two TFNs with $\alpha$-cut $\widetilde{A}_{1}^{\alpha}=\left[a_{1}+\right.$ $\left.\alpha \cdot\left(b_{1}-a_{1}\right), c_{1}-\alpha \cdot\left(c_{1}-b_{1}\right)\right]$ and $\widetilde{A}_{2}^{\alpha}=\left[a_{2}+\alpha \cdot\left(b_{2}-a_{2}\right), c_{2}-\alpha \cdot\left(c_{2}-b_{2}\right)\right]$, respectively. If $a_{1}+\alpha \cdot\left(b_{1}-a_{1}\right) \leq a_{2}+\alpha \cdot\left(b_{2}-a_{2}\right)$ and $\left.\left.c_{1}-\alpha \cdot\left(c_{1}-b_{1}\right)\right] \leq c_{2}-\alpha \cdot\left(c_{2}-b_{2}\right)\right]$, then

$$
F_{Q}\left(\widetilde{A}_{1}^{\alpha}\right) \leq F_{Q}\left(\widetilde{A}_{2}^{\alpha}\right)
$$

Proof. Denoting $A_{i}^{L}=a_{i}+\alpha \cdot\left(b_{i}-a_{i}\right)$ and $A_{i}^{U}=c_{i}-\alpha \cdot\left(c_{i}-b_{i}\right)$, we have

$$
F_{Q}\left(\widetilde{A}_{2}^{\alpha}\right)-F_{Q}\left(\widetilde{A}_{1}^{\alpha}\right)=(1-\lambda) \cdot\left(A_{2}^{L}-A_{1}^{L}\right)+\lambda \cdot\left(A_{2}^{U}-A_{1}^{U}\right)
$$

If $A_{1}^{L} \leq A_{2}^{L}$ and $A_{1}^{U} \leq A_{2}^{U}$ then it is $F_{Q}\left(\widetilde{A}_{2}^{\alpha}\right)-F_{Q}\left(\widetilde{A}_{1}^{\alpha}\right) \geq 0$.

In the following we introduce the attitudinal expected score function of an TFN:

Definition 9 (TFN Attitudinal Expected Score Function). Given a BMU function $Q$ with attitudinal character value $\lambda$, the attitudinal expected score degree of a TFN $\widetilde{A}=(a, b, c)$ is

$$
A E S_{\lambda}(\widetilde{A})=2 \cdot \int_{0}^{1} F_{Q}\left(\widetilde{A}^{\alpha}\right) \alpha d \alpha=\frac{(1-\lambda) a+2 b+\lambda c}{3} .
$$


The following properties hold:

Proposition 4 (Additivity Property). Let $\widetilde{A}_{1}=\left(a_{1}, b_{1}, c_{1}\right)$ and $\widetilde{A}_{2}=\left(a_{2}, b_{2}, c_{2}\right)$ be two TFNs then $A E S_{\lambda}\left(\widetilde{A}_{1}+\widetilde{A}_{2}\right)=A E S_{\lambda}\left(\widetilde{A}_{1}\right)+A E S_{\lambda}\left(\widetilde{A}_{2}\right)$.

Proof. Proof of Proposition4 is a consequence of Proposition 2.

Proposition 5 (Monotonocity). Let $\widetilde{A}_{1}=\left(a_{1}, b_{1}, c_{1}\right)$ and $\widetilde{A}_{2}=\left(a_{2}, b_{2}, c_{2}\right)$ be two TFNs with $\alpha$-cut $\widetilde{A}_{1}^{\alpha}$ and $\widetilde{A}_{2}$, respectively. If $F_{Q}\left(\widetilde{A}_{1}^{\alpha}\right) \leq F_{Q}\left(\widetilde{A}_{2}^{\alpha}\right) \forall \alpha$, then $A E S_{\lambda}\left(\widetilde{A}_{1}\right) \leq A E S_{\lambda}\left(\widetilde{A}_{2}\right)$

Proof. Proof is trivial and therefore it is omitted.

The attitudinal expected score function can be used to define an ordering relation on a given set of TFNs. However, this ordering is much dependent on the BUM function reflecting the attitude of moderator. Indeed, a change on the value of $\lambda$ could result in a different ordering of two ITFNs, as the following example illustrates.

Example 8. The following two TFNs $\widetilde{A}_{1}=(0.5,0.7,0.9)$ and $\widetilde{A}_{2}=(0.6,0.7,0.8)$ have the following attitudinal expected score values, $A E S_{\lambda}\left(\widetilde{A}_{1}\right)=\frac{1.9+0.4 \lambda}{3}$ and $A E S_{\lambda}\left(\widetilde{A}_{2}\right)=\frac{2.0+0.2 \lambda}{3}$, respectively. Their ranking depends on the moderator's attitudinal character as follows:

1. $A E S_{\lambda}\left(\widetilde{A}_{1}\right)<A E S_{\lambda}\left(\widetilde{A}_{2}\right)$ if and only if $\lambda>0.5$

2. $A E S_{\lambda}\left(\widetilde{A}_{1}\right)=A E S_{\lambda}\left(\widetilde{A}_{2}\right)$ if and only if $\lambda=0.5$

3. $A E S_{\lambda}\left(\widetilde{A}_{1}\right)>A E S_{\lambda}\left(\widetilde{A}_{2}\right)$ if and only if $\lambda<0.5$

$\mathrm{Xu}$ [67] introduced a sensitivity analysis for interval fuzzy preference relation by the COWA operator. In the following we will provide a sensitivity analysis of the attitudinal expected score function with respect to the attitudinal character $\lambda$. In addition, we will also provide the conditions under which the ordering of two TFNs is not affected by a change in the attitudinal parameter.

Let $\lambda$ be the attitudinal parameter associated to the BUM function $Q$ under which it has been established that $A E S_{\lambda}\left(\widetilde{A}_{i}\right)<A E S_{\lambda}\left(\widetilde{A}_{j}\right)$. Assume that the attitudinal parameter is perturbed by a quantity $\Delta \lambda$ to become $\lambda+\Delta \lambda$. Let us denote by $A E S_{\lambda+\Delta \lambda}\left(\widetilde{A}_{i}\right)$ and $A E S_{\lambda+\Delta \lambda}\left(\widetilde{A}_{j}\right)$ the new attitudinal expected score degrees. Then, one question is presented as: what are the conditions $\Delta \lambda$ needs to verify so that $A E S_{\lambda+\Delta \lambda}\left(\widetilde{A}_{i}\right) \leq A E S_{\lambda+\Delta \lambda}\left(\widetilde{A}_{j}\right)$, i.e. the ranking of TFNs does not change? The following theorem provides the answer to this question.

Theorem 1. Let $\widetilde{A}_{i}$ and $\widetilde{A}_{j}$ be two TFNs with expected score degrees such that $A E S_{\lambda}\left(\widetilde{A}_{i}\right)<$ $A E S_{\lambda}\left(\widetilde{A}_{j}\right)$. Let $\Delta \lambda$ be a perturbation of the attitudinal character $\lambda$ with $0 \leq \lambda+\Delta \lambda \leq 1$. Then 
we have $A E S_{\lambda+\Delta \lambda}\left(\widetilde{A}_{i}\right)<A E S_{\lambda+\Delta \lambda}\left(\widetilde{A}_{j}\right)$ if and only if

$$
\begin{cases}\max \left\{-\lambda, \frac{3 \cdot\left[A E S_{\lambda}\left(\widetilde{A}_{j}\right)-A E S_{\lambda}\left(\widetilde{A}_{i}\right)\right]}{\beta_{i}-\beta_{j}}\right\} \leq \Delta \lambda \leq 1-\lambda, & \beta_{i}<\beta_{j} \\ -\lambda \leq \Delta \lambda \leq 1-\lambda, & \beta_{i}=\beta_{j} \\ -\lambda \leq \Delta \lambda \leq \min \left\{1-\lambda, \frac{3 \cdot\left[A E S_{\lambda}\left(\widetilde{A}_{j}\right)-A E S_{\lambda}\left(\widetilde{A}_{i}\right)\right]}{\beta_{i}-\beta_{j}}\right\}, & \beta_{i}>\beta_{j}\end{cases}
$$

where $\beta_{i}=c_{i}-a_{i}$ and $\beta_{j}=c_{j}-a_{j}$.

Proof. Firstly, we note that $\Delta \lambda$ is subject to the following constraint: $-\lambda \leq \Delta \lambda \leq 1-\lambda$. We have the following relation between $A E S_{\lambda}\left(\widetilde{A}_{i}\right)$ and $A E S_{\lambda+\Delta \lambda}\left(\widetilde{A}_{i}\right)$ :

$$
A E S_{\lambda+\Delta \lambda}\left(\widetilde{A}_{i}\right)=A E S_{\lambda}\left(\widetilde{A}_{i}\right)+\frac{\Delta \lambda \cdot \beta_{i}}{3}
$$

where $\beta_{i}=c_{i}-a_{i}$. The following equivalence holds:

$$
A E S_{\lambda+\Delta \lambda}\left(\widetilde{A}_{i}\right)<A E S_{\lambda+\Delta \lambda}\left(\widetilde{A}_{j}\right) \Leftrightarrow \Delta \lambda \cdot\left(\beta_{i}-\beta_{j}\right)<3 \cdot\left[A E S_{\lambda}\left(\widetilde{A}_{j}\right)-A E S_{\lambda}\left(\widetilde{A}_{i}\right)\right]
$$

Three scenarios are possible:

- $\beta_{i}=\beta_{j}$. Because $A E S_{\lambda}\left(\widetilde{A}_{j}\right)-A E S_{\lambda}\left(\widetilde{A}_{i}\right)>0$ then (34) is true for any value of $\Delta \lambda$ subject to $-\lambda \leq \Delta \lambda \leq 1-\lambda$.

- $\beta_{i}>\beta_{j} \Leftrightarrow \Delta \lambda<\frac{3 \cdot\left[A E S_{\lambda}\left(\widetilde{A}_{j}\right)-A E S_{\lambda}\left(\widetilde{A}_{i}\right)\right]}{\beta_{i}-\beta_{j}}$, and therefore:

$$
-\lambda \leq \Delta \lambda \leq \min \left\{1-\lambda, \frac{3 \cdot\left[A E S_{\lambda}\left(\widetilde{A}_{j}\right)-A E S_{\lambda}\left(\widetilde{A}_{i}\right)\right]}{\beta_{i}-\beta_{j}}\right\} .
$$

- $\beta_{i}<\beta_{j} \Leftrightarrow \Delta \lambda>\frac{3 \cdot\left[A E S_{\lambda}\left(\widetilde{A}_{j}\right)-A E S_{\lambda}\left(\widetilde{A}_{i}\right)\right]}{\beta_{i}-\beta_{j}}$, and therefore:

$$
\max \left\{-\lambda, \frac{3 \cdot\left[A E S_{\lambda}\left(\widetilde{A}_{j}\right)-A E S_{\lambda}\left(\widetilde{A}_{i}\right)\right]}{\beta_{i}-\beta_{j}}\right\} \leq \Delta \lambda \leq 1-\lambda .
$$

\subsection{Attitudinal prioritisation and ranking sensitivity analysis of TFCPRs}

The application of the attitudinal expected score function to each one of the TFNs of a TFCPR allows us to derive a numerical fuzzy preference relation (FPR) that we call the attitudinal score FPR (AS-FPR): 
Definition 10. (Attitudinal Score FPR (AS-FPR)) Let $\widetilde{P}=\left(\widetilde{p}_{i k}\right)_{n \times n}$ be a TFCPR and $\lambda$ an attitudinal parameter value, then we call $S P^{\lambda}=\left(s p_{i k}^{\lambda}\right)_{n \times n}$ the attitudinal score FPR corresponding to $\widetilde{P}$,where

$$
s p_{i k}=A E S_{\lambda}\left(\widetilde{p}_{i k}^{\lambda}\right), \quad s p_{k i}=1-A E S_{\lambda}\left(\widetilde{p}_{i k}^{\lambda}\right) \quad \forall i<k .
$$

Using expression (32) we have:

$$
s p_{i k}^{\lambda}=\left((1-\lambda) a_{i k}+2 b_{i k}+\lambda c_{i k}\right) / 3, \quad s p_{k i}^{\lambda}=1-s p_{i k} \quad \forall i<k
$$

Using the AS-FPR $S P^{\lambda}$ associated to a given TFCPR $\widetilde{P}$, we can define the attitudinal expected preference degree of an alternative $x_{i}$ over the rest alternatives as follows:

$$
s p_{i}^{\lambda}=\frac{1}{n} \sum_{k=1}^{n} s p_{i k}^{\lambda}=\frac{\sum_{k=1}^{n}\left[(1-\lambda) a_{i k}+2 b_{i k}+\lambda c_{i k}\right]}{3 n}
$$

The attitudinal expected preference degrees can be used to produce a final ranking of the alternatives and the fore to facilitate the selection of the final solution of consensus to the GDM problem with TFCPRs, as the following example illustrates.

Example 9. (Example 1 continuation) For the collective TFCPR $\widehat{P}$, suppose that the following BUM function $Q(y)=y^{3}$ represents the moderator's attitudinal character. The attitudinal character $\lambda$ would be

$$
\lambda=\int_{0}^{1} Q(y) d y=\int_{0}^{1} y^{3} d y=\frac{1}{4} .
$$

The AS-FPR associated to the collective TFCPR $\widehat{P}$ is

$$
S P^{0.25}=\left(\begin{array}{cccc}
- & 0.493 & 0.513 & 0.613 \\
0.507 & - & 0.593 & 0.323 \\
0.487 & 0.407 & - & 0.473 \\
0.387 & 0.677 & 0.527 & -
\end{array}\right)
$$

The attitudinal expected preference degrees associated to each alternative would be:

$$
p_{1}^{0.25}=0.530, p_{2}^{0.25}=0.481, p_{3}^{0.25}=0.467, p_{4}^{0.25}=0.523
$$

which results in the following final ordering

$$
x_{1} \succ x_{4} \succ x_{2} \succ x_{3} .
$$

Again, the ordering of alternatives depends on the attitudinal BUM function. To obtain the conditions under which the ordering of two alternatives is affected by a change in the attitude of moderator, we will present a sensitivity analysis with respect to the attitudinal character $\lambda$. 
Theorem 2. Let $\widetilde{P}=\left(\widetilde{p}_{i j}\right)$ be an TFCPR and $S P^{\lambda}=\left(s p_{i j}^{\lambda}\right)$ be its associated $A S-F P R$ such that $s p_{i}^{\lambda}<s p_{j}^{\lambda}$. Let $\Delta \lambda$ be perturbation of the attitudinal character $\lambda$ with $0 \leq \lambda+\Delta \lambda \leq 1$. Then we have

$$
s p_{i}^{\lambda+\Delta \lambda}<s p_{j}^{\lambda+\Delta \lambda} \Leftrightarrow \begin{cases}\max \left\{-\lambda, \frac{3 n \cdot\left(s p_{j}^{\lambda}-s p_{i}^{\lambda}\right)}{\delta_{i}-\delta_{j}}\right\} \leq \Delta \lambda \leq 1-\lambda, & \text { if } \delta_{i}<\delta_{j} \\ -\lambda \leq \Delta \lambda \leq 1-\lambda, & \text { if } \delta_{i}=\delta_{j} \\ -\lambda \leq \Delta \lambda \leq \min \left\{1-\lambda, \frac{3 n \cdot\left(s p_{j}^{\lambda}-s p_{i}^{\lambda}\right)}{\delta_{i}-\delta_{j}}\right\}, & \text { if } \delta_{i}>\delta_{i}\end{cases}
$$

where $\delta_{i}=\sum_{k=1}^{n}\left(c_{i k}-a_{i k}\right)$ and $\delta_{j}=\sum_{k=1}^{n}\left(c_{j k}-a_{j k}\right)$.

Proof. On the one hand, we have that $\Delta \lambda$ is subject to the following constraint: $-\lambda \leq \Delta \lambda \leq 1-\lambda$. On the other hand, we have

$$
p s_{i}^{\lambda+\Delta \lambda}=s p_{i}^{\lambda}+\frac{\Delta \lambda \cdot \delta_{i}}{3 n}
$$

where $\delta_{i}=\sum_{k=1}^{n}\left(c_{i k}-a_{i k}\right)$. The following equivalence holds:

$$
s p_{i}^{\lambda+\Delta \lambda}<s p_{j}^{\lambda+\Delta \lambda} \Leftrightarrow \Delta \lambda \cdot\left(\delta_{i}-\delta_{j}\right)<3 n \cdot\left(s p_{j}^{\lambda}-s p_{i}^{\lambda}\right)
$$

- If $\delta_{i}=\delta_{j}$, then because $s p_{i}^{\lambda}<s p_{j}^{\lambda}$ we have that expression (38) is always true, and therefore

$$
-\lambda \leq \Delta \lambda \leq 1-\lambda
$$

- If $\delta_{i}>\delta_{j}$ then $\Delta \lambda \leq \frac{3 n \cdot\left(s p_{j}^{\lambda}-s p_{i}^{\lambda}\right)}{\delta_{i}-\delta_{j}}$, and therefore

$$
-\lambda \leq \Delta \lambda \leq \min \left\{1-\lambda, \frac{3 n \cdot\left(s p_{j}^{\lambda}-s p_{i}^{\lambda}\right)}{\delta_{i}-\delta_{j}}\right\} .
$$

- If $\delta_{i}<\delta_{j}$ then $\Delta \lambda \geq \frac{3 n \cdot\left(s p_{j}^{\lambda}-s p_{i}^{\lambda}\right)}{\delta_{i}-\delta_{j}}$, and therefore:

$$
\max \left\{-\lambda, \frac{3 n \cdot\left(s p_{j}^{\lambda}-s p_{i}^{\lambda}\right)}{\delta_{i}-\delta_{j}}\right\} \leq \Delta \lambda \leq 1-\lambda .
$$

The following example provides an analysis of the ordering of the alternatives of the previous example based on the value of the attitudinal parameter $\lambda$.

Example 10. (Finishing Example 1) Applying expression (37), the attitudinal expected preference degrees associated to each alternative would be

$$
s p_{1}^{\lambda}=\frac{47+6 \cdot \lambda}{120}, \quad s p_{2}^{\lambda}=\frac{42+2 \cdot \lambda}{120}, s p_{3}^{\lambda}=\frac{41-2 \cdot \lambda}{120}, s p_{4}^{\lambda}=\frac{49-6 \lambda}{120} .
$$


It is easy to get that

$$
\begin{array}{lll}
s p_{1}^{\lambda}>s p_{2}^{\lambda} & \text { if and only if } & 0 \leq \lambda \leq 1, \\
s p_{1}^{\lambda}>s p_{4}^{\lambda} & \text { if and only if } & 0.175<\lambda \leq 1, \\
s p_{2}^{\lambda}>s p_{3}^{\lambda} & \text { if and only if } & 0 \leq \lambda \leq 1, \\
s p_{2}^{\lambda}>s p_{4}^{\lambda} & \text { if and only if } & 0.875<\lambda \leq 1, \\
s p_{4}^{\lambda}>s p_{3}^{\lambda} & \text { if and only if } & 0 \leq \lambda \leq 1,
\end{array}
$$

Then, we obtain the following ranking scenarios based on the value of the attitudinal parameter $\lambda$ :

$$
\begin{aligned}
& x_{4} \succ x_{1} \succ x_{2} \succ x_{3} \text { if } 0 \leq \lambda<0.175 \\
& x_{4} \sim x_{1} \succ x_{2} \succ x_{3} \text { if } \lambda=0.175 \\
& x_{1} \succ x_{4} \succ x_{2} \succ x_{3} \text { if } 0.175 \leq \lambda<0.875 \\
& x_{1} \succ x_{4} \sim x_{2} \sim x_{3} \text { if } \lambda=0.875 \\
& x_{1} \succ x_{2} \succ x_{4} \succ x_{3} \text { if } 0.875<\lambda \leq 1
\end{aligned}
$$

Obviously, the ordering of the alternatives is susceptible to a change in a value of $\lambda$. Additionally, an optimistic moderator will tend to select alternative $x_{1}$, while a pessimistic one will choose alternative $x_{4}$. Hence, our approach is able to rank the alternatives by taking into account attitudinal character of the group of experts or its moderator if applicable.

\section{Analysis of the GDM model}

This proposed methods for GDM problems with TFCPRs presents the following main advantages with respect to other consensus models proposed in the literature:

1. It includes a visual information feedback mechanism following a top to bottom methodology to find the discordant opinion, provide recommendations to those experts that are furthest from the group, and automatically simulate future consensus status if experts are to follow recommendations in clear pictures. Therefore, the advantage of our proposed model is that it dose not force decision makers (DMs) to change their opinions, but provide more information to help DMs to revisit their decision.

2. It allows the presence of TFCPRs, which captures uncertainty more appropriately than numeric preference relations or ordinal linguistic preference relations. It is important to remark that the proposed model is one of the first efforts in introducing TFCPRs into the field of consensus GDM.

3. It investigates a COWA operator based prioritisation method for TFCPRs. The novelty of this method is that it can rank the alternatives according to the attitudinal character of moderator.

4. It presents a sensitivity analysis for the final ranking order of the alternatives with respect to the attitudinal parameter, providing flexibility in its adaptation to different scenarios both in terms 
of uncertainty modelling and pessimistic or optimistic approach to solve the decision making problem.

Also, the proposed GDM method still exhibits some limitations to be addressed in future research.

1. In most cases, group consensus facilitates the acceptance of the decision outcome. However, it is meaningless when most experts of the group provide incorrect information. Therefore, how to determine the presence of incorrect information is an interesting problem that has not been successfully addressed and/or implemented by any previous consensus GDM models.

2. Although this paper investigates a COWA operator based prioritisation method by taking into account the attitudinal character, including a sensitivity analysis for the moderator, it may be difficult to determine his/her attitude directly in real decision cases. We plan to carry out future research to develop a linguistic sentiment scale and apply sentiment analysis to determine the attitudinal character of the moderator.

\section{Conclusion}

In this paper, a novel visual information feedback mechanism for GDM problems with TFCPRs has been presented. To achieve this, the concepts of similarity degree (SD) between two experts as well as the proximity degree (PD) between an experts and the rest of experts in the group are developed for TFCPRs. These degrees are used to compute both the aggregation weighting vector as well as the consensus level of the group of experts. The visual information feedback mechanism is investigated to identify experts, alternatives and corresponding preference values that contribute less to consensus. Recommendations to help experts the direction of the change required to increase their consensus are produced and an automatic visual feedback process simulation to show the experts what would happen if they were to follow recommendations by pictures is developed. Finally, a new prioritisation method of alternatives for a TFCFPR based on the implementation of the attitudinal character is presented. Furthermore, a ranking sensitivity analysis of the attitudinal expected score function with respect to the attitudinal parameter is addressed and solved. Summarising, the presented consensus model supports the aggregation of TFCPRs in GDM to achieve solutions with high degree of consensus that reflect in a correct way the attitudinal character desired to be implemented, and consequently provides further flexibility to decision making under uncertainty in inconsistent environments.

\section{Acknowledgements}

The authors thank for the constructive suggestions from the anonymous referees. This work was supported by National Natural Science Foundation of China (NSFC) under the Grant No.71101131 and No.713311002, Humanities and Social Science Projects of Chinese Ministry of Education under 
the Grant No.10YJC630277, and Zhejiang Provincial National Science Foundation for Distinguished Young Scholars of China under the Grant No. LR13G010001.

\section{References}

[1] S. Alonso, F. Chiclana, F. Herrera, E. Herrera-Viedma. A learning procedure to estimate missing values in fuzzy preference relations based on additive consistency. Lecture Notes Artificial Intelligence3131 (2004) 227-238.

[2] S. Alonso, F. Chiclana, F. Herrera, E. Herrera-Viedma, J. Alcala-Fdez, C. Porcel. A consistencybased procedure to estimate missing pairwise preference values. International Journal of Intelligent Systems 23 (2008) 155-175.

[3] S. Alonso, E. Herrera-Viedma, F. Chiclana, F. Herrera. A web based consensus support system for group decision making problems and incomplete preferences. Information Sciences 180 (2010) $4477-4495$.

[4] A. Altuzarra, J. M. Moreno-Jiménez, M. Salvador. Consensus Building in AHP-Group Decision Making: A Bayesian Approach. Operations Research 58 (2010) 1755-1773.

[5] A. Arbel. Approximate articulation of preference and priority derivation. European Journal of Operational Research 43 (1989) 317-326.

[6] D. Ben-Arieh, Z. F. Chen. Linguistic-labels aggregation and consensus measure for autocratic decision making using group recommendations. IEEE Transactions on Systems, Man, and Cybernetics - Part A: Systems and Humans 36 (2006) 558-568.

[7] N. Bryson. Group decision-making and the analytic hierarchy process: Exploring the consensusrelevant information content Computers $\& 3$ Operations Research 23 (1996) 27-35.

[8] P. Burillo, H. Bustince. Entropy on intuitionistic fuzzy sets and on interval-valued fuzzy sets. Fuzzy Sets and Systems 78(3) (1996) 305-316.

[9] S. M. Chen, L. W. Lee. Autocratic Decision Making Using Group Recommendations Based on the ILLOWA Operator and Likelihood-Based Comparison Relations. IEEE Transactions on Systems, Man, and Cybernetics - Part A: Systems and Humans 42(1) (2012) 115-129.

[10] F. Chiclana, F. Herrera, E. Herrera-Viedma. Integrating three representation models in fuzzy multipurpose decision making based on fuzzy preference relations. Fuzzy Sets and Systems 97(1) (1998) 33-48. 
[11] F. Chiclana, F. Herrera, E. Herrera-Viedma. Integrating multiplicative preference relations in a multipurpose decision-making model based on fuzzy preference relations. Fuzzy Sets and Systems 122(2) (1998) 277-291.

[12] F. Chiclana, E. Herrera-Viedma, F. Herrera, S. Alonso. Some induced ordered weighted averaging operators and their use for solving group decision-making problems based on fuzzy preference relations. European Journal of Operational Research 182 (2007) 383-399.

[13] F. Chiclana, E. Herrera-Viedma, S. Alonso, F. Herrera. Cardinal consistency of reciprocal preference relations: a characterization of multiplicative transitivity. IEEE Transactions on Fuzzy Systems 17(1) (2009) 14-23.

[14] F. Chiclana, J. M. Tapia-Garcia, M. J. del Moral, E. Herrera-Viedma. A statistical comparative study of different similarity measures of consensus in group decision making. Information Sciences 221 (2013) 110-123.

[15] M. Dagdeviren, I. Yuksel. Developing a fuzzy analytic hierarchy process (AHP) model for behavior-based safety management. Information Sciences 178 (2008) 1717-1733.

[16] M. M. Deza, E. Deza. Encyclopedia of Distances. Springer Berlin Heidelberg. 2009.

[17] Y. C. Dong, Y. F Xu, H. Li, B. Feng. An overview on the 2-tuple linguistic model for computing with words in decision making: Extensions, applications and challenges. European Journal of Operational Research 203 (2010) 455-463.

[18] P. Eklund, A. Rusinowska, H. de Swart. Y. C. Dong, Y. F Xu, H. Li, B. Feng. A consensus model of political decision-making. Annals of Operations Research 158 (2008) 5-20.

[19] Z. P. Fan, Y. Liu. An approach to solve group decisionmaking problems with ordinal interval numbers. IEEE Transactions on Systems, Man, and Cybernetics, Part B: Cybernetics 40 (2005) $1413-1423$.

[20] M. Fedrizzi, S. Giove. Incomplete pairwise comparison and consistency optimization. European Journal of Operational Research 183 (2007) 303-313.

[21] P. C. Fishburn. The Theory of Social Choice. Princeton University Press. 1973.

[22] P. C. Fishburn. Utility Theory for Decision Making. Robert E. Krieger Publishing Company. 1979 .

[23] C. Fu, S. L. Yang. The group consensus based evidential reasoning approach for multiple attributive group decision analysis. European Journal of Operational Research 206 (2010) 601-608. 
[24] S. Genc, F. E. Bo, D. Akay, Z. S. Xu. Interval multiplicative transitivity for consistency, missing values and priority weights of interval fuzzy preference relations. Information Sciences 180 (2010) $4877-4891$.

[25] S. Greenfield, F. Chiclana, S. Coupland, R. I. John. The collapsing method of defuzzification for discretised interval type-2 fuzzy sets. Information Sciences 179(13) (2009) 2055-2069.

[26] S. Greenfield, F. Chiclana, S. Coupland, R. I. John. The Sampling Method of Defuzzification for Type-2 Fuzzy Sets: Experimental Evaluation. Information Sciences 189 (2012) 77-92.

[27] F. Herrera, S. Alonso, F. Chiclana, E. Herrera-Viedma. Computing with words in decision making: foundations, trends and prospects. Fuzzy Optimization and Decision Making 8 (2009) 337-364.

[28] F. Herrera, E. Herrera-Viedma, J. L. Verdegay. Direct approach processes in group decision making using linguistic OWA operators. Fuzzy Sets and Systems 79 (1996) 175-190.

[29] F. Herrera, E. Herrera-Viedma F. Chiclana. Multiperson decision-making based on multiplicative preference relations. European Journal of Operational Research 129(1) (2001) 372-385.

[30] F. Herrera, L. Martinez. A 2-tuple fuzzy linguistic representation model for computing with words. IEEE Transactions on Fuzzy Systems 8 (2000) 746-752.

[31] E. Herrera-Viedma, F. Herrera, F. Chiclana. A consensus model for multiperson decision making with different preference structures. IEEE Transactions on Systems, Man, and Cybernetics - Part A: Systems and Humans 32 (2002) 394-402.

[32] E. Herrera-Viedma, F. Herrera, F. Chiclana, M. Luque. Some issues on consistency of fuzzy preference relations. European Journal of Operational Research 154(1) (2004) 98-109.

[33] E. Herrera-Viedma, F. Chiclana, F. Herrera, S. Alonso. Group Decision-Making Model With Incomplete Fuzzy Preference Relations Based on Additive Consistency. IEEE Transactions on Systems, Man, and Cybernetics, Part B: Cybernetics 37(1) (2007a) 176-189.

[34] E. Herrera-Viedma, S. Alonso, F. Chiclana, F. Herrera. A Consensus Model for Group Decision Making With Incomplete Fuzzy Preference Relations. IEEE Transactions on Fuzzy Systems 15(5) (2007b) 863-877.

[35] H. M. Hsu, C T. Chen. Aggregation of fuzzy opinions under group decision making. Fuzzy Sets and Systmes 79 (1996) 279-285.

[36] J. Kacprzyk, M. Fedrizzi, H. Nurmi. A concise consensus support model for group decision making with reciprocal preference relations based on deviation measures. Fuzzy Sets and Systems $49(1992) 21-31$. 
[37] J. Kacprzyk, S. Zadrozny, Z. W. Ras. How to support consensus reaching using action rules: a novel approach. International Journal of Uncertainty, Fuzziness and Knowledge-Based Systems 36 (2010) 451-470.

[38] P. J. M. Laarhoven, W. Pedrycz. A fuzzy extension of Saaty's priority theory. Fuzzy Sets Systems 11 (1983) 229-241.

[39] F. Liu, W. G Zhang, Z. X. Wang. A goal programming model for incomplete interval multiplicative preference relations and its application in group decision-making. European Journal of Operational Research 218 (2012a) 747-754.

[40] F. Liu, W. G. Zhang, J. H. Fu. A new method of obtaining the priority weights from an interval fuzzy preference relation. Information Sciences 185 (2012b) 32-42.

[41] J. Ma, Z. P Fan, Y. P. Jiang, J. Y. Mao, Ma. L. A method for repairing the inconsistency of reciprocal relations. Fuzzy Sets and Systems 157 (2006) 20-33.

[42] L. Martinez, F. Herrera. An overview on the 2-tuple linguistic model for computing with words in decision making: Extensions, applications and challenges. Information Sciences 207 (2012) 1-18.

[43] F. Mata, L. Martinez, E. Herrera-Viedma. An adaptive consensus support model for group decision-making problems in a multigranular fuzzy linguistic context. IEEE Transaction on Fuzzy Systems 17 (2009) 279-290.

[44] G. H. Marakas. Decision support systems in the 21th century. Upper Saddle River, NJ: Pearson Education, 2003.

[45] I. Millet. The effectiveness of alternative preference elicitation methods in the analytic hierarchy process. Journal of Multi-Criteria Decision Analysis 6 (1997) 41-51.

[46] R. O. Parreiras, P. Y. Ekel, J. S. C. Martini, R. M. Palhares. A flexible consensus scheme for multicriteria group decision making under linguistic assessments. Information Sciences 180 (2010) 1075-1089.

[47] T. L. Saaty. The analytic hierarchy process. New York: McGraw-Hill. 1980.

[48] T. Tanino. Fuzzy preference orderings in group decision making. Fuzzy sets and system 12 (1984) $117-131$.

[49] F. Tiryaki, B. Ahlatcioglu. Fuzzy portfolio selection using fuzzy analytic hierarchy process. Information Sciences 179 (2009) 53-69.

[50] E. Turban and J. E. Aronsos. Decision support systems and intelligent systems. Upper Saddle River, NJ: Prentice-Hall, 2001. 
[51] C. T. Wang, Y. H. Chen. Applying fuzzy linguistic preference relations to the improvement of consistency of fuzzy AHP. Information Sciences 178 (2008) 3755-3765.

[52] Y. M. Wang, J. B. Yang, D. L. Xu. A two-stage logarithmic goal programming method for generating weights from interval comparison matrices. Fuzzy Sets and Systems 152 (2005) 475498.

[53] Y. M. Wang, K, S. Chin. A linear goal programming priority method for fuzzy analytic hierarchy process and its applications in new product screening. International Journal of Approximate Reasoning 49 (2008) 451-465.

[54] Y. M. Wang, Y. Luo, Z, S. Hua. On the extent analysis method for fuzzy AHP and its applications. European Journal of Operational Research 186 (2008) 735-747.

[55] Z. J. Wang, K. W. Li. Goal programming approaches to deriving interval weights based on interval fuzzy preference relations. Information Sciences 193 (2005) 180-198.

[56] J. Wu, Q. W. Cao. Some issues on properties of the extended IOWA operators in fuzzy group decision making. Expert Systems with Applications 38(2011) 7059-7066.

[57] J. Wu, Q. W. Cao, J. L. Zhang. Some properties of the induced continuous ordered weighted geometric operators in group decision making. Computers and Industrial Engineering 59 (2010) $100-106$.

[58] J. Wu, Q. W. Cao, J. L. Zhang. An ILOWG operator based group decision making method and its application to evaluate the supplier criteria. Mathematical and Computer Modelling 54 (2011) $19-34$.

[59] J. Wu, F. Chiclana Non-dominance and attitudinal prioritisation methods for intuitionistic and interval-valued intuitionistic fuzzy preference relations. Expert Systems with Applications 39 (2012) 13409-13416.

[60] J. Wu, J. C. Li, H. Li, W. Q. Duan. The induced continuous ordered weighted geometric operators and their application in group decision making. Computers and Industrial Engineering 57 (2009) $1545-1552$.

[61] Z. B. Wu, J. P. Xu. A concise consensus support model for group decision making with reciprocal preference relations based on deviation measures. Fuzzy Sets and Systems 206 (2012a) 58-73.

[62] Z. B. Wu, J. P. Xu A consistency and consensus based decision support model for group decision making with multiplicative preference relations. Decision Support Systems 52 (2012b) 757-767. 
[63] M. M Xia, Z. S. Xu. Methods for fuzzy complementary preference relations based on multiplicative consistency. Computers and Industrial Engineering 61 (2011) 930-935.

[64] Y. J. Xu, R. Patnayakuni, H. M. Wang. The ordinal consistency of a fuzzy preference relation. Information Sciences 224 (2013) 152-164.

[65] Z. S. Xu. On consistency of the weighted geometric mean complex judgement matrix in AHP. European Journal of Operational Research 126 (2000) 683-687.

[66] Z. S. Xu. Deviation measures of linguistic preference relations in group decision making. Omega 33 (2005) 249-254.

[67] Z. S. Xu. A C-OWA operator-based approach to decision making with interval fuzzy preference relation. International Journal of Intelligent Systems 21 (2006) 1289-1298.

[68] Z. S. Xu. A survey of preference relations. International Journal of General System 27(36) (2007) 179-203.

[69] Z. S. Xu. Group decision making based on multiple types of linguistic preference relations. Information Sciences 178 (2008) 452-467.

[70] Z. S. Xu, X. Q. Cai. Group consensus algorithms based on preference relations. Information Science 181 (2011) 150-162.

[71] R. R. Yager. Owa aggregation over a continuous interval argument with applications to decision making. IEEE Transactions on Systems, Man, and Cybernetics, Part B: Cybernetics 34(5) (2004) $1952-1963$.

[72] R. R. Yager, Z. S. Xu. The continuous ordered weighted geometric operator and its application to decision making. Fuzzy Sets and Systems 157 (2006) 1393-1402.

[73] L. A. Zadeh. Fuzzy sets. Information and Control 8(3) (1965) 338-357.

[74] L. A. Zadeh. The concept of a linguistic variable and its application to approximate reasoning-I. Information Sciences 8 (1975) 199-249.

[75] L. A. Zadeh. The concept of a linguistic variable and its application to approximate reasoning-II. Information Sciences 8 (1975) 301-357.

[76] F. Zahedi. Group Consensus Function Estimation When Preferences are Uncertain. Operations research $34(6)$ (1986) 883-894.

[77] R. Zwick, E. Carlstein, D. V. Budescu. Measures of similarity among fuzzy concepts: A comparative analysis. International Journal of Approximate Reasoning 1 (1987) 221-242. 